\title{
INEQUALITIES FOR SUMS OF INDEPENDENT RANDOM VARIABLES
}

\author{
N. L. CAROTHERS AND S. J. DILWORTH \\ (Communicated by William J. Davis)
}

\begin{abstract}
A moment inequality is proved for sums of independent random variables in the Lorentz spaces $L_{p, q}$, thus extending an inequality of Rosenthal. The latter result is used in combination with a square function inequality to give a proof of a Banach space isomorphism theorem. Further moment inequalities are also proved.
\end{abstract}

1. Introduction. First some notation and standard terminology. Let $(M, \Sigma, m)$ be a measure space. For $1<p<\infty$ and $0<q \leq \infty$ the Lorentz space $L_{p, q}(M)$ is the collection of all measurable $f$ on $M$ such that

$$
\begin{gathered}
\|f\|_{p, q}=\left(\frac{q}{p} \int_{0}^{\infty} f^{*}(t)^{q} t^{q / p-1} d t\right)^{1 / q}<\infty \quad(0<q<\infty) \\
\|f\|_{p, \infty}=\underset{t>0}{\operatorname{ess} \sup } t^{1 / p} f^{*}(t)<\infty
\end{gathered}
$$

where $f^{*}$ denotes the decreasing rearrangement of $|f|$. Note that $L_{p, p}(M)$ is just the Lebesgue space $L_{p}(M)$ and that $L_{p, \infty}$ is the space weak- $L_{p}$. The $L_{p, q}$ spaces arise in the Lions-Peetre $K$-method of interpolation: in particular,

$$
L_{p, q}=\left[L_{p_{1}}, L_{p_{2}}\right]_{\theta, q}, \quad \text { where } 1 / p=(1-\theta) / p_{1}+\theta / p_{2}
$$

Standard facts about $L_{p, q}$ and the $K$-method will be used throughout; these may be found in, for example, [13 and 1].

To avoid awkward formulae we reserve the letter $C$ to denote a positive constant (whose precise value may change from line to line) depending only on the parameters $p, q$, etc. We write $A \sim B$ when there exists such a constant $C$ with $(1 / C) A \leq$ $B \leq C A$.

In [11] Rosenthal proved the following moment inequality for independent symmetric random variables defined on a probability space $(\Omega, \mathcal{F}, P)$.

Received by the editors June 1, 1987 and, in revised form, September 15, 1987.

1980 Mathematics Subject Classification (1985 Revision). Primary 46E30, 60G50; Secondary 46B25, 60B11.

Key words and phrases. Lorentz function spaces, sums of independent random variables, LionsPeetre interpolation.

The second author was supported in part by NSF DMS-8500764. 
THEOREM. Given $2<p<\infty$ there exists a constant $C$, depending only on $p$, such that

(1)

$$
\begin{aligned}
\max \left(\left\|\sum_{k=1}^{n} X_{k}\right\|_{2},\left(\sum_{k=1}^{n}\left\|X_{k}\right\|_{p}^{p}\right)^{1 / p}\right) & \leq\left\|\sum_{k=1}^{n} X_{k}\right\|_{p} \\
& \leq C \max \left(\left\|\sum_{k=1}^{n} X_{k}\right\|_{2},\left(\sum_{k=1}^{n}\left\|X_{k}\right\|_{p}^{p}\right)^{1 / p}\right)
\end{aligned}
$$

for all independent symmetric random variables $X_{1}, X_{2}, \ldots, X_{n}$ in $L_{p}(\Omega)$.

In this paper we give some extensions and applications of Rosenthal's inequality. In $\S 2$ we prove an analogue for the $L_{p, q}$ norm of a sum of independent symmetric random variables. In $\S 3$ we show how (1) can be used in conjunction with a square function inequality of Stein to give a short proof of an isomorphism result from [8]. The paper concludes with some further consequences of Stein's inequality.

Finally, we should like to thank Bill Johnson and Joel Zinn for several enlightening conversations about Rosenthal's inequality, and Gideon Schechtman for showing us the short proof of Proposition 3.1.

2. Extension to $L_{p, q}$. Given a measurable function $f$ on a measure space $(M, \Sigma, m)$ define its distribution $d_{f}$ by $d_{f}(t)=m(\{|f| \geq t\})$, and given random variables $f_{1}, f_{2}, \ldots, f_{n}$ on the probability space $\Omega$ define the disjoint sum, denoted $\sum_{k=1}^{n} \bigoplus f_{k}$, to be any function $f$ on $(0, \infty)$ with $d_{f}(t)=\sum_{k=1}^{n} d_{f_{k}}(t)$. Recall also that the intersection of two Lorentz spaces $L_{p_{1}, q_{1}}(0, \infty)$ and $L_{p_{2}, q_{2}}(0, \infty)$ is a quasi-Banach space under the quasi-norm $\max \left(\|f\|_{p_{1}, q_{1}},\|f\|_{p_{2}, q_{2}}\right)$.

LEMMA 2.1. Let $0<r<s<\infty, 0<\theta<1$, and $0<q \leq \infty$. Then

$$
\left[L_{r}, L_{r} \cap L_{s}\right]_{\theta, q}=L_{r} \cap\left[L_{r}, L_{s}\right]_{\theta, q}
$$

where $L_{p}$ denotes $L_{p}(0, \infty)$ throughout.

PROOF. It is easily seen that $\left[L_{r}, L_{r} \cap L_{s}\right]_{\theta, q} \subset L_{r} \cap\left[L_{r}, L_{s}\right]_{\theta, q}$. To show the reverse inclusion fix $f \in L_{r} \cap\left[L_{r}, L_{s}\right]_{\theta, q}$ and let $K(t)=K\left(t, f ; L_{r}, L_{r} \cap L_{s}\right)$. Then for $t \geq 1, K(t) \leq\|f\|_{r}=K\left(t, f ; L_{r}, L_{r}\right)$. For $t<1$ we use Holmstedt's formula [7]: set $1 / \alpha=1 / r-1 / s$, then

$$
\begin{aligned}
K(t) & \leq\left\|f^{*} I\left(0, t^{\alpha}\right)\right\|_{r}+t\left\|f^{*} I\left(t^{\alpha}, \infty\right)\right\|_{L_{r} \cap L_{s}} \\
& \leq\left\|f^{*} I\left(0, t^{\alpha}\right)\right\|_{r}+t\left\|f^{*} I\left(t^{\alpha}, \infty\right)\right\|_{s}+t\|f\|_{r} \\
& \sim K\left(t, f ; L_{r}, L_{s}\right)+K\left(t, f ; L_{r}, L_{r}\right) .
\end{aligned}
$$

The desired inclusion now follows easily. 
THEOREM 2.2. Given $2<p<\infty$ and $0<q \leq \infty$, there exists a constant $C$ (depending only on $p$ and $q$ ) such that

(2)

$$
\begin{aligned}
\frac{1}{C} \max \left(\left\|\sum_{k=1}^{n} X_{k}\right\|_{2},\left\|\sum_{k=1}^{n} \bigoplus X_{k}\right\|_{p, q}\right) & \leq\left\|\sum_{k=1}^{n} X_{k}\right\|_{p, q} \\
& \leq C \max \left(\left\|\sum_{k=1}^{n} X_{k}\right\|\left\|_{2},\right\| \sum_{k=1}^{n} \bigoplus X_{k} \|_{p, q}\right)
\end{aligned}
$$

for all independent symmetric random variables $X_{1}, X_{2}, \ldots, X_{n}$ in $L_{p, q}(\Omega)$.

PROOF. It is convenient to take $\Omega$ to be $[0,1]^{\mathbf{N}}$ with the product measure and to denote a typical element of $\Omega$ by the sequence $s=\left(s_{1}, s_{2}, \ldots\right)$. First observe that the theorem simply reduces to Rosenthal's inequality in the case $p=q$. Define a linear operator $T: L_{0}(0, \infty) \rightarrow L_{0}(\Omega \times[0,1])$ by $T(f)=\sum_{k=1}^{\infty} f_{k}\left(s_{k}\right) r_{k}(t)$, where $f_{k}\left(s_{k}\right)=f\left(k-1+s_{k}\right)$ and $r_{k}(t)$ is the $k$ th Rademacher function. Then by (1) $T$ is a bounded operator (in fact an isomorphic embedding) from $L_{2}(0, \infty) \cap L_{p}(0, \infty)$ into $L_{p}(\Omega \times[0,1])$ for $p>2$. So by Lemma 2.1 and Marcinkiewicz interpolation $T$ is bounded from $L_{2}(0, \infty) \cap L_{p, q}(0, \infty)$ into $L_{p, q}(\Omega \times[0,1])$, which proves the right-hand inequality of $(2)$. Now

$$
\left\|\sum_{k=1}^{n} X_{k}\right\|_{2} \leq C\left\|\sum_{k=1}^{n} X_{k}\right\|_{p, q}
$$

since $p>2$, and so to prove the left-hand inequality it is enough to show that

$$
\left\|\sum_{k=1}^{n} \oplus X_{k}\right\|_{p, q} \leq C\left\|\sum_{k=1}^{n} X_{k}\right\|_{p, q} .
$$

Since $\sum_{k=1}^{n} X_{k}$ has the same distribution as $\sum_{k=1}^{n} X_{k}(s) r_{k}(t)$, by the generalization of Khintchine's inequality to lattices [10, Theorems 1.d.6 and 2.b.11] it is enough to show that

$$
\left\|\sum_{k=1}^{n} \oplus X_{k}\right\|_{p, q} \leq C\left\|\left(\sum_{k=1}^{n} X_{k}^{2}\right)^{1 / 2}\right\|_{p, q},
$$

and this follows from [5, Corollary 2.7] for $p>2$.

REMARK 2.3. Further inequalities for sums of random variables in $L_{p, q}$ are proved in [4]. These are used in [5] to study the subspace structure of $L_{p, q}(0, \infty)$. The problem of interpolating between arbitrary intersections of $L_{p}$ spaces along the lines of Lemma 2.1 is solved in [6].

3. Further results. Let $Y_{p}$ denote the space $L_{p}(0, \infty) \cap L_{2}(0, \infty)$ for $2 \leq p \leq \infty$ and $L_{p}(0, \infty)+L_{2}(0, \infty)$ for $0<p \leq 2$. Note that

$$
\|f\|_{Y_{p}} \sim\left\|f^{*} I(0,1)\right\|_{p}+\left\|f^{*} I(1, \infty)\right\|_{2}
$$

and that $\left(Y_{p}\right)^{*}=Y_{q}$ if $p \geq 1$ and if $1 / p+1 / q=1$. Using the operator $T$ of Theorem 2.2 we give a proof of the beautiful theorem from [8] which states that $Y_{p}$ and $L_{p}(0,1)$ are isomorphic Banach spaces for $1<p<\infty$. This is a rather 
different approach from that of [8], in which a stochastic integral with respect to a symmetrized Poisson process is used to embed $Y_{p}$ into $L_{p}$. We need the following square function inequality, which follows easily from a result of Stein. It is also implicit in a result of Bryc and Kwapien [2].

PROPOSITION 3.1. Let $1<p<\infty$ and let $\left(\mathcal{F}_{k}\right)_{k=1}^{n}$ be independent sub- $\sigma$-fields of $\mathcal{F}$. There exists $C_{p}$ such that

$$
\left\|\left(\sum_{k=1}^{n}\left(E\left(f_{k} \mid \mathcal{F}_{k}\right)\right)^{2}\right)^{1 / 2}\right\|_{p} \leq C_{p}\left\|\left(\sum_{k=1}^{n} f_{k}^{2}\right)^{1 / 2}\right\|_{p}
$$

for all $n \geq 1$ and for all $f_{1}, f_{2}, \ldots, f_{n}$ in $L_{p}(\Omega)$.

PROOF. This is a theorem of Stein [12] when $\left(\mathcal{F}_{k}\right)_{k=1}^{n}$ is replaced by an increasing sequence $\left(\mathcal{G}_{k}\right)_{k=1}^{n}$ of sub- $\sigma$-fields. Let $\mathcal{G}_{k}$ and $\widetilde{\mathscr{G}}_{k}$ be the sub- $\sigma$-fields generated by $\bigcup_{i=1}^{k} \mathcal{F}_{i}$ and $\bigcup_{i=n-k}^{n} \mathcal{F}_{i}$, respectively. Applying Stein's square function inequality first with respect to $\left(\mathcal{G}_{k}\right)_{k=1}^{n}$ and then with respect to $\left(\widetilde{\mathcal{G}}_{k}\right)_{k=1}^{n}$ gives the result.

THEOREM 3.2. $Y_{p}$ and $L_{p}(0,1)$ are isomorphic for $1<p<\infty$.

PROOF. Take $\Omega$ to be $[0,1]^{\mathrm{N}}$ as in Theorem 2.2 and identify $\mathcal{F}$ with the sub- $\sigma$ field $\{F \times[0,1]: F \in \mathcal{F}\}$ of the product $\sigma$-field in $\Omega \times[0,1]$. It was observed in the proof of Theorem 2.2 that the operator $T: Y_{p} \rightarrow L_{p}(\Omega \times[0,1])$ is an isomorphic embedding for $2 \leq p<\infty$. Define $P: L_{p}(\Omega \times[0,1]) \rightarrow L_{p}(\Omega \times[0,1])$ by

$$
P(f)=\sum_{k=1}^{\infty} E\left(f r_{k} \mid s_{k}\right) r_{k}(t)
$$

(recall that $s_{1}, s_{2}, \ldots$ are the independent coordinates of an element $s$ in $\Omega$ ). Then $P$ is a selfadjoint projection onto the range of $T$. To see that $P$ is bounded for $1<p<\infty$, note that by Khintchine's inequality and Proposition 3.1 we have

$$
\begin{aligned}
\|P f\|_{p} & \sim\left\|\left(\sum_{k=1}^{\infty}\left(E\left(f r_{k} \mid s_{k}\right)\right)^{2}\right)^{1 / 2}\right\|_{p} \\
\leq & C_{p}\left\|\left(\sum_{k=1}^{\infty}\left(E\left(f r_{k} \mid \mathcal{F}\right)\right)^{2}\right)^{1 / 2}\right\|_{p} \\
& \sim\left\|\sum_{k=1}^{\infty} E\left(f r_{k} \mid \mathcal{F}\right) r_{k}\right\|_{p} .
\end{aligned}
$$

But

$$
\left\|\sum_{k=1}^{\infty} E\left(f r_{k} \mid \mathcal{F}\right) r_{k}\right\|_{p} \leq C_{p}\|f\|_{p}
$$

for $1<p<\infty$ : this is just the conditional form of the well-known fact that the Rademacher sequence spans a complemented subspace of $L_{p}$. Since $P$ is selfadjoint it follows that $P$ is a bounded projection onto $Y_{p}$ (i.e. the range of $T$ ) for all 
$1<p<\infty$. Since $L_{p}(0,1)$ is plainly isomorphic to a complemented subspace of $Y_{p}$, Pełczýnski's decomposition argument (e.g. [10]) will complete the proof.

The existence of the projection $P$ in the last result implies the following extension of Rosenthal's inequality to the range $1<p<2$; this extension was first proved by Johnson and Schechtman [9].

COROLlary 3.3. Let $1<p<2$. Then

$$
\left\|\sum_{k=1}^{n} X_{k}\right\|_{p} \sim\left\|\sum_{k=1}^{n} \bigoplus X_{k}\right\|_{Y_{p}}
$$

for all independent symmetric random variables $X_{1}, X_{2}, \ldots, X_{n}$.

A different proof of this result will appear in [9] together with its generalization to the setting of rearrangement invariant spaces.

We conclude with some further consequences of Proposition 3.1.

COROLlaRY 3.4. Let $1<p \leq \infty$ and let $\left(\mathcal{F}_{k}\right)_{k=1}^{n}$ be independent sub- $\sigma$-fields

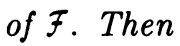

$$
\left\|\left(\sum_{k=1}^{n}\left(E\left(f \mid \mathcal{F}_{k}\right)\right)^{2}\right)^{1 / 2}\right\|_{p} \leq C_{p} \sqrt{n}\|f\|_{p}
$$

for all $f \in L_{p}(\Omega)$.

PROOF. Take $f_{k}=f(1 \leq k \leq n)$ in Proposition 3.1 in the range $1<p<$ $\infty$. The case $p=\infty$ is obvious because the conditional expectation operator is a contraction on $L_{\infty}(\Omega)$.

REMARK 3.5 . Observe that the choice of $f \equiv 1$ shows that the constant $\sqrt{n}$ is of the correct order. Corollary 3.4 is only interesting in the range $1<p<2$, for in the range $2 \leq p<\infty$ it follows easily from Minkowski's inequality. The result breaks down completely when $p=1$. To see this, let $X_{1}, X_{2}, \ldots, X_{n}$ be independent nonnegative random variables with $E X_{k}=1$. Then $E\left(\prod_{i=1}^{n} X_{i} \mid X_{k}\right)=$ $X_{k}$ and $\left\|\prod_{k=1}^{n} X_{k}\right\|_{1}=1$. Taking $X_{k}=\left|\theta_{k}\right|$, where $\theta_{1}, \theta_{2}, \ldots, \theta_{n}$ are independent symmetric $r$-stable random variables $(1<r<2)$, gives $\left\|\left(\sum_{k=1}^{n} X_{k}^{2}\right)^{1 / 2}\right\|_{1} \sim n^{1 / r}$. So when $p=1$ the factor of $\sqrt{n}$ in Corollary 3.4 must be replaced by $n$.

Our final theorem should be viewed as a generalization of Khintchine's inequality.

THEOREM 3.6. Let $1<p<2$ and let $X_{1}, X_{2}, \ldots, X_{n}$ be independent symmetric random variables. Then

$$
\frac{\left\|\sum_{k=1}^{n} X_{k}\right\|_{p}}{\prod_{k=1}^{n}\left\|X_{k}\right\|_{p}} \leq \frac{C_{p}\left(\sum_{k=1}^{n}\left\|X_{k}\right\|_{1}^{2}\right)^{1 / 2}}{\prod_{k=1}^{n}\left\|X_{k}\right\|_{1}}
$$

ProOF. Let $a_{1}, a_{2}, \ldots, a_{n}$ be real numbers. Applying Proposition 3.1 with $f_{k}=a_{k} f(1 \leq k \leq n)$ gives

$$
\left\|\left(\sum_{k=1}^{n}\left(a_{k} E\left(f \mid \mathcal{F}_{k}\right)\right)^{2}\right)^{1 / 2}\right\|_{p} \leq C_{p}\left(\sum_{k=1}^{n} a_{k}^{2}\right)^{1 / 2}\|f\|_{p} .
$$

Let

$$
X=\prod_{k=1}^{n} \frac{\left|X_{k}\right|}{\left\|X_{k}\right\|_{1}}
$$


Then

$$
\|X\|_{p}=\prod_{k=1}^{n} \frac{\left\|X_{k}\right\|_{p}}{\left\|X_{k}\right\|_{1}}
$$

and

$$
E\left(X \mid X_{k}\right)=\frac{\left|X_{k}\right|}{\left\|X_{k}\right\|_{1}}
$$

Putting $f=X$ and $a_{k}=\left\|X_{k}\right\|_{1}$ gives

$$
\left\|\left(\sum_{k=1}^{n} X_{k}^{2}\right)^{1 / 2}\right\|_{p} \leq C_{p}\left(\sum_{k=1}^{n}\left\|X_{k}\right\|_{1}^{2}\right)^{1 / 2} \frac{\prod_{k=1}^{n}\left\|X_{k}\right\|_{p}}{\prod_{k=1}^{n}\left\|X_{k}\right\|_{1}}
$$

while by Khintchine's inequality

$$
\left\|\sum_{k=1}^{n} X_{k}\right\|_{p} \sim\left(\sum_{k=1}^{n} X_{k}^{2}\right)^{1 / 2} \|_{p}
$$

The desired conclusion now follows.

\section{REFERENCES}

1. J. Bergh and J. Löfstrom, Interpolation spaces, Grundlehren Math. Wiss., vol. 223, SpringerVerlag, 1976.

2. W. Bryc and S. Kwapien, On the conditional expectation with respect to a sequence of independent $\sigma$-fields, Z. Wahrsch. Verw. Gebiete 46 (1979), 221-225.

3. N. L. Carothers and S. J. Dilworth, Geometry of Lorentz spaces via interpolation, "Longhorn Notes", Univ. of Texas Functional Analysis Seminar, 1985-86.

4. __ Equidistributed random variables in $L_{p, q}$, J. Funct. Anal. (to appear).

5. __ Subspaces of $L_{p, q}$, Proc. Amer. Math. Soc. (to appear).

6. S. J. Dilworth, Interpolation of intersections of $L_{p}$ spaces, Arch. Math. 50 (1988), 51-55.

7. T. Holmstedt, Interpolation of quasi-normed spaces, Math. Scand. 26 (1970), 177-199.

8. W. B. Johnson, B. Maurey, G. Schechtman and L. Tzafriri, Symmetric structures in Banach spaces, Mem. Amer. Math. Soc. no. 217, 1979.

9. W. B. Johnson and G. Schechtman, Sums of independent random variables in rearrangement invariant function spaces, preprint (1986).

10. J. Lindenstrauss and L. Tzafriri, Classical Banach spaces. II. Function spaces, Springer-Verlag, 1979.

11. H. P. Rosenthal, On the subspaces of $L_{p}(p>2)$ spanned by sequences of independent random variables, Israel J. Math. 8 (1970), 273-303.

12. E. M. Stein, Topics in harmonic analysis related to the Littlewood-Paley theory, Ann. of Math. Studies, no. 63, Princeton Univ. Press, Princeton, N. J., 1970.

13. E. M. Stein and G. Weiss, Introduction to Fourier analysis on Euclidean spaces, Princeton Univ. Press, Princeton, N.J., 1971.

Department of Mathematics, Bowling Green State University, Bowling GREEN, OHIO 43403

Department of Mathematics, University of South Carolina, Columbia, SOUTH CAROLINA 29208 